\title{
Maturitätsprofile als differenzielle Lernkontexte. Eine empirische Analyse des Zusammen- hanges zwischen institutionellen Rahmun- gen und Kompetenzentwicklungen der Gymnasiastinnen und Gymnasiasten.
}

\section{Katharina Maag Merki}

In diesem Beitrag wird die Frage gestellt, inwiefern in der gymnasialen Ausbildung in der Entwicklung von überfachlichen Kompetenzen sowohl profil- wie auch geschlechtsspezifische Effekte in Abhängigkeit des Lernkontextes (Maturitätsprofile) sichtbar werden. Die präsentierten Ergebnisse basieren auf einer $2 \times 2$ univariaten multifaktoriellen Kovarianzanalyse. Die Ergebnisse zeigen, dass Maturitätsprofile als Institutionalisierung schulischer Ausbildungen in einem bedeutsamen Zusammenhang stehen mit der Entwicklung der überfachlichen Kompetenzen der Schüler/innen. Interaktionseffekte lassen sich in einzelnen Kompetenzdimensionen identifizieren und verweisen in den meisten Fällen darauf, dass sich männliche Jugendlichen in einzelnen Profilen stärker von ibren Kollegen in den anderen Profilen unterscheiden als weibliche Jugendliche.

Die Sekundarstufe II stellt für die berufliche oder universitäre Ausbildung einen bedeutsamen Abschnitt in der Bildungsbiographie der Jugendlichen dar. Sie erweist sich insbesondere aus einer geschlechtsspezifischen Perspektive als besonders bedeutsam, da in dieser Stufe nach der obligatorischen Schulzeit über die Wahl der weiterführenden Schulen oder der beruflichen Ausbildung spezifische moderierende Effekte sichtbar werden, die die in der beruflichen Arbeitswelt nach Abschluss der Ausbildung festzustellenden geschlechtsspezifischen Segregationen vorspuren (Eccles, 2005; Gildemeister \& Wetterer, 1992). Im Gegensatz zur Sekundarstufe I, die in verschiedensten umfangreichen Studien bereits häufig untersucht worden ist (z.B. PISA, LAU, Konstanzer Längsschnittstudie), liegen bis anhin auf der Sekundarstufe II nur wenige Studien vor, die die Kompetenzentwicklung der Schüler/innen in Abhängigkeit individueller und institutioneller Faktoren untersucht haben. Zudem fokussieren diese Studien vor allem die fachspezifische Kompetenzentwicklung und sind meist querschnittlich angelegt (vgl. Baumert, Bos \& Lehmann 2000; Baumert et al., 1996; Köller, Watermann \& Trautwein, 2004; Lehmann, Hunger, Ivanova, Gänsfuss \& Hoffmann, 2004). Gerade für die Sekundarstufe II fehlen damit Studien, in denen nicht fachspezifische Kompetenzen in ihrer längsschnittlichen Entwicklung 
untersucht worden sind. Wird der Fokus auf Kompetenzbereiche gelegt, die nicht den traditionellen fachlichen Disziplinen folgen, so ermöglicht dies, das Kompetenzprofil von jungen Frauen und Männern differenzierter zu beschreiben.

In diesem Beitrag wird die Frage gestellt, inwiefern auf der Sekundarstufe II - in dieser Studie beschränkt auf die gymnasiale Ausbildung - in der Entwicklung von überfachlichen Kompetenzen (Grob \& Maag Merki, 2001) sowohl profilspezifische wie auch geschlechtsspezifische Effekte in Abhängigkeit des Lernkontextes (Maturitätsprofile) sichtbar werden. Diese Analysestrategie ist in dem Sinne interessant, als die Maturitätsprofile institutionalisierte Ausbildungskonfigurationen mit je spezifischen Profilbildungen und Lehrplänen darstellen. So führt die Wahl eines Profils zu unterschiedlichen Fächerkombinationen, wobei einzelne Fächer in der Stundentafel der einzelnen Profile ein unterschiedliches Gewicht haben. Die Maturitätsprofile sind damit als Regulative menschlichen Handelns (Gildemeister \& Wetterer, 1992) zu verstehen, wobei Studienergebnisse darauf hinweisen, dass das Ausmass an aktiver Lernzeit für die Auseinandersetzung mit fachlichen Inhalten ein wichtiger Faktor zur Vorhersage von Schulleistungen darstellt (Helmke \& Weinert, 1997). Die Wahl eines bestimmten Ausbildungskontextes durch die Schüler/innen ist einerseits geprägt durch die Interessen an bestimmten Fächern (Ramseier, Allraum \& Stadler, 2005). Andererseits kann diese Wahl durch weitere Faktoren bestimmt sein, so beispielsweise durch die Erfolgserwartung im zukünftigen Studium/Beruf, die Möglichkeiten, im jeweiligen Studium/Beruf eigene Ziele zu erreichen, die potenziellen Kosten, die zu investieren sind oder die kulturell geprägten Rollenschemata, wobei hier sowohl geschlechtsspezifische wie auch ethnische oder soziale Kategorien als Basis dienen können (Eccles, 2005, 2006; Filipp, 2006, S. 66). Die ungleiche Verteilung der Geschlechter in den verschiedenen Maturitätsprofilen in den Gymnasien und einzelne empirische Ergebnisse (Ramseier, Allraum \& Stadler 2005) deuten darauf hin, dass auch in der gymnasialen Ausbildung geschlechtsspezifisch geprägte Prozesse und Entscheidungsmuster bei der Wahl von Maturitätsprofilen wirksam sind.

Auch wenn davon auszugehen ist, dass die Wahl der Maturitätsprofile bereits mit einem spezifischen Kompetenzprofil der Schüler/innen in einem Zusammenhang steht, so ist doch unklar, welche Effekte diese Lernkonfigurationen im Verlauf der Schuljahre auf die Entwicklung überfachlicher Kompetenzen für alle Schüler/innen eines Profils und für die einzelnen Teilgruppen (Schülerinnen vs. Schüler) ausüben. So lässt sich fragen, inwiefern junge Frauen (bzw. junge Männer) in den spezifischen Lernkontexten unterschiedliche Verläufe aufweisen werden als ihre Kolleginnen (bzw. Kollegen) in den anderen Lernkontexten. Die Ergebnisse aus Studien zum Zusammenhang von fachlichen Leistungen und gymnasialen Schultypen (Watermann, Nagy \& Köller, 2004) sowie aus Studien der Arbeitswelt (Heintz, Nadai, Fischer \& Ummel, 1997) lassen vermuten, dass 
die Lernkontexte nicht nur im fachlichen Bereich, sondern auch bei den überfachlichen Kompetenzen einen bedeutsamen Einfluss ausüben und der Entwicklungsverlauf für männliche und weibliche Jugendliche in männer- oder frauendominierten Lernkonfigurationen zumindest teilweise unterschiedlich ausgestaltet ist. So zeigen Untersuchungen in der Arbeitswelt (Heintz et al., 1997), dass die Entwicklungen von sogenannten "go-betweens», von Frauen und Männern, die die beruflichen Grenzen überschritten haben und in einem Beruf arbeiten, der traditionellerweise in der Mehrheit vom anderen Geschlecht gewählt wird, d.h. Frauen in «Männerberufen» (z.B. Informatikerin) bzw. Männer in «Frauenberufen» (z.B. Krankenpfleger), divergent zu jenen ihrer Kolleginnen und Kollegen in den traditionellen Berufen sind. Die Ergebnisse verweisen darauf, dass Männer in frauenspezifischen Berufen und Frauen in männerspezifischen Berufen unterschiedlich mit dieser Situation umgehen und unterschiedliche Erfahrungen der Integration bzw. Segregation machen. Zudem zeigen die Ergebnisse ebenfalls, dass das Umfeld unterschiedlich auf die Minderheitssituation reagiert, beispielsweise in dem Sinne, als Männer in frauenspezifischen Berufen speziell von ihren Vorgesetzten gefördert und von den Frauen integriert werden, während dem Frauen in männerdominierten Berufen stärker mit Ausgrenzung und Diskriminierung konfrontiert werden. Zu untersuchen ist damit, ob es neben den profilspezifischen Effekten auch spezifische Interaktionseffekte zwischen dem gewählten Profil und dem Geschlecht geben wird.

\section{Gymnasium, Geschlecht und überfachliche Kompetenzen}

Die gymnasiale Ausbildung in der Schweiz gehört aufgrund historischer Entwicklungen zu denjenigen Bildungsstufen, die am längsten geschlechtsspezifisch geprägt worden sind, beispielsweise durch die späte Zulassung von Mädchen zum Gymnasium oder die während langer Zeit vorgefundene Trennung von Mädchen- und Jungengymnasien. Heute wird nicht nur die Mehrheit der Gymnasien koedukativ geführt. Vielmehr lässt sich seit mehreren Jahrzehnten feststellen, dass zunehmend mehr weibliche Jugendliche das Gymnasium besuchen und dieses mit der Maturität abschliessen (1980: 42.5\%; 2004: 57.4\%)1. Zudem ist die Häufigkeit des Scheiterns beim Maturitätsabschluss bei den weiblichen Jugendlichen niedriger als bei den Gymnasiasten (Ramseier, Allraum \& Stadler, 2005). Analoge Entwicklungen finden sich auch für den Kanton Zürich, in dem die hier präsentierte Studie durchgeführt worden ist. ${ }^{2}$

Als zentrale Zielkategorien schulischer Bildung werden im Maturitätanerkennungsreglement MAR überfachliche Kompetenzen formuliert ((Schweizerischer Bundesrat \& Erziehungsdirektorenkonferenz EDK, 1995; Grob \& Maag Merki, 2001; Grob, Maag Merki \& Büeler, 2003). Sie lassen sich als fachübergreifende, aber auf Situationen und Inhalte bezogene individuelle Dispositionen definie- 
ren, die die Wahrscheinlichkeit erhöhen, anspruchsvollen Anforderungen in Schule, Beruf und Gesellschaft gerecht zu werden und sie mit Erfolg zu bewältigen. Neben kognitiven Aspekten schliesst das Konzept auch motivationale, moralische, volitionale und soziale Komponenten ein (vgl. dazu auch Weinert, 2001). Damit werden neben Wissensstrukturen explizit auch Einstellungen, Selbstkonzepte oder motivationale Orientierungen als überfachliche Kompetenzen bezeichnet. Im Gegensatz zum Intelligenzkonzept wird davon ausgegangen, dass (überfachliche) Kompetenzen durch Erfahrung und Lernen sowie durch äussere Interventionen sukzessive angeeignet, aufgebaut bzw. adaptiv modifiziert werden können.

Bis anhin fehlen Studien, in denen überfachliche Kompetenzen im Gymnasium auf der Sekundarstufe II umfassend erfasst worden sind. Es gibt jedoch Studien, die einzelne Kompetenzdimensionen auf der Sekundarstufe II oder auf anderen Schulstufen untersucht haben. Allerdings werden in einem grossen Teil dieser Studien einzig individuelle geschlechtsspezifische Effekte ohne Berücksichtigung allfälliger institutioneller Effekte untersucht (z.B. personale Kompetenzen: Block \& Robins, 1993, Horstkemper, 1991; gesundheitsbezogene Kompetenzen: Bundesamt für Statistik, 2003, Hurrelmann, Klocke, Melzer \& Ravens-Sieberer, 2003; Lernkompetenzen: Artelt, Baumert, Julius-McElvany \& Peschar, 2003, Brühlwiler \& Biedermann, 2005; gesellschaftsbezogene Kompetenzen: Bieri \& Forrer, 2005; Fend, 2000).

Zur Analyse institutioneller Effekte liegen Studienergebnisse insbesondere im Bereich der Lernkompetenzen und punktuell im Bereich der sozialen Kompetenzen vor. So wurde im Rahmen der TOSCA-Studie (Köller et al., 2004) untersucht, inwiefern bestimmte Schultypen auf der gymnasialen Oberstufe neben dem Geschlecht einen Einfluss auf den Einsatz verschiedener wissenschaftsnaher Lern- und Arbeitsformen haben (Trautwein \& Lüdtke, 2004). Zusätzlich zu geschlechtsspezifischen Effekten auf individueller Ebene zeigen sich signifikante Effekte aufgrund des gewählten Schultyps im Bereich der Informationsbeschaffung (z.B. Informationen für Arbeiten finden, Literatur zusammenstellen), in der Gestaltung von Referaten, bei der Computernutzung, in der Planung von Experimenten und im Anfertigen von Protokollen. Diese Effekte sind in der Computernutzung (besonders ausgeprägt in Technischen Gymnasien und im Agrarwissenschaftlichen Gymnasium) und in der Planung von Experimenten (besonders ausgeprägt im Technischen, Ernährungswissenschaftlichen und Agrarwissenschaftlichen Gymnasium) besonders gross. Als möglicher Einflussfaktor wird der Besuch unterschiedlicher Leistungskurse durch die Schüler/innen erwähnt. Bei den anderen Kompetenzdimensionen steht die grosse Variabilität im Antwortverhalten nur in einem schwachen Zusammenhang mit dem besuchten Schultyp (vgl. ebd., S. 349).

Hinweise auf systematische Unterschiede in Abhängigkeit der Maturitätstypen liegen für die Lernmotivation im Fach Mathematik im Rahmen der TIMSSStudie vor (Ramseier, 2004, S. 201). Es besteht ein signifikanter Unterschied in 
der intrinsischen Orientierung zwischen den Schüler/innen des auf Mathematik und Naturwissenschaften ausgerichteten Maturitätstypus $\mathrm{C}$ und den übrigen Typen. Zudem zeigt sich in der Tendenz, dass die männlichen Jugendlichen im Typus $C$ stärker intrinsisch motiviert sind als die weiblichen Jugendlichen in Typus C. Dieser Befund findet sich in den anderen Maturitätstypen nicht. Bei der langfristig-instrumentellen Orientierung sind sowohl der Geschlechtsunterschied zugunsten der Männer als auch der Unterschied zwischen den Typen hoch signifikant und erheblich. Frauen scheinen in der Mathematik einen geringeren Nutzen als Männer zu sehen und wählen daher eher weniger den Typus C.

Institutionelle Effekte zeigen sich auch in der Studie von Eder (1996). Zum einen wurde das allgemeine Selbstwertgefühl der Schüler/innen in Abhängigkeit des Schultyps - berufsbildende vs. allgemeinbildende Schulen - untersucht. Dabei hat sich gezeigt, dass es bei den jungen Männern vom 9. bis zum 13. Schuljahr zu einer deutlichen Steigerung der positiven Selbstwahrnehmung kommt, während dieser Effekt bei den jungen Frauen in den allgemeinbildenden Schulen viel langsamer und in den berufsbildenden Schulen überhaupt ausbleibt (ebd., S. 186). Hinsichtlich des Entwicklungsverlaufes des sozialen Selbstkonzeptes der Jugendlichen zeigen sich ebenfalls differenzielle Effekte da sich die Differenzen zwischen den Geschlechtern von der 9. bis zur 12. Jahrgangsstufe der allgemeinbildenden höheren Schulen zunehmend verringern. Ein Unterschied zugunsten der jungen Frauen, der in vielen Querschnittstudien belegt ist (z.B. Ford, 1982; Gensicke, 2002; Stanat \& Kunter, 2001) ist somit kaum mehr bedeutsam. In den berufsbildenden höheren Schulen weisen die jungen Männer im 13. Schuljahr sogar höhere Werte auf als die jungen Frauen.

\section{Fazit}

Insgesamt liegen einzelne Studien vor, die institutionelle Effekte, auch in Abhängigkeit des Geschlechts in einzelnen Kompetenzbereichen untersucht haben, wobei der Fokus auf einzelne Dimensionen der Lernkompetenz (Lernstrategien und Lernmotivation) sowie auf einzelne personale und soziale Kompetenzen (Selbstwert bzw. soziales Selbstkonzept) gelegt worden ist. Hingegen fehlen Studien, die diese Effekte auf der Basis von längsschnittlichen Daten und in weiteren Kompetenzbereichen (z.B. gesellschaftsbezogene oder gesundheitsbezogene Kompetenzen) unter einer vergleichenden Perspektive analysiert haben.

Aufgrund der bisherigen Ergebnisse (Eder, 1996; Ramseier, 2004; Trautwein \& Lüdtke, 2004; Heintz et al., 1997) kann angenommen werden, dass es zumindest in einzelnen Kompetenzdimensionen institutionelle Effekte gibt, die einen systematischen Zusammenhang zwischen der Entwicklung der Kompetenzen der Jugendlichen und den institutionellen Kontexten erwarten lassen (These 1). Zudem sind auch Interaktionseffekte zu vermuten, die auf einen geschlechtsspezifischen Entwicklungsverlauf in Abhängigkeit des Lernkontextes bzw. des gewählten Maturitätsprofils hinweisen (These 2). Entsprechend den Analysen von Heintz et al. (1997) kann vermutet werden, dass es in der Ausprä- 
gung der differenziellen Effekte Unterschiede zwischen den jungen Frauen und jungen Männern geben wird (These 2a), wobei diese in einem Zusammenhang stehen mit dem Geschlechterverhältnis im jeweiligen Maturitätsprofil (These $2 \mathrm{~b})$. Insgesamt sind aufgrund bisheriger empirischer Ergebnisse insbesondere Effekte bei den Lernkompetenzen und einzelnen personalen und sozialen Kompetenzen zu erwarten (These 3). Aufgrund der fehlenden empirischen Grundlage für gesellschafts- und gesundheitsbezogene Kompetenzen können hierzu keine Hypothesen formuliert, sondern einzig explorative Analysen vorgenommen werden.

\section{Methodisches Verfahren}

Die vorliegenden Analysen sind Teil eines längsschnittlich angelegten Forschungsvorhabens in der Schweiz, in dem in allen öffentlichen $(\mathrm{N}=20)$ und zwei privaten Gymnasien im Kanton Zürich alle Schüler/innen eines ausgewählten Schuljahrganges hinsichtlich der Entwicklung ihrer überfachlichen Kompetenzen zu zwei verschiedenen Zeitpunkten untersucht worden sind (Maag Merki, 2006a). Der erste Erhebungszeitpunkt wurde auf Anfang des 10. Schuljahres 2001 festgesetzt. Als zweiter Erhebungszeitpunkt wurde das Ende der gymnasialen Ausbildung im Jahre 2004 gewählt. Die Schüler/innen standen zu diesem Zeitpunkt kurz vor den Abschlussprüfungen (Maturität bzw. Abitur). Zur Untersuchungsgruppe wurden nur diejenigen Schüler/innen gezählt, die sich an beiden Erhebungen beteiligt hatten. Der drop-out bewirkte keine systematischen Verzerrungen der Untersuchungsgruppe, so dass die Analysen ohne Gewichtungen vorgenommen werden können (Maag Merki \& Leutwyler, 2004). Die Verteilung der Schüler/innen nach Geschlecht und Maturitätsprofil findet sich in Tabelle 1.

Zur Erfassung der überfachlichen Kompetenzen wird ein standardisiertes Instrumentarium eingesetzt, das sich bereits in mehreren Studien als reliabel und valide erwiesen hat (Bieri \& Forrer, 2005; Grob \& Maag Merki, 2001). Die einzelnen Konstrukte werden auf der Basis von selbstbezogenen Kognitionen erfasst (mit Ausnahme zweier Skalen zur Erfassung des Wissensstands im Bereich Politik und Umwelt, die mit Hilfe von Raschmodellierungen entwickelt worden sind und Wissensfragen beinhalten). Dabei wird davon ausgegangen, dass selbstbezogene Kognitionen in einer bedeutsamen Beziehung zu den jeweiligen überfachlichen Kompetenzen und den entsprechenden Handlungsweisen stehen (Epstein, 1993; Filipp, 2006; Filipp, 1993; Greve, 2000). 
Tabelle 1: Maturitätsprofile im Kanton Zürich; Veteilung in der Untersuchungsgruppe

\begin{tabular}{|l|c|c|c|c|}
\hline & \multicolumn{2}{|c|}{ Gesamt } & Junge Frauen & Junge Männer \\
\hline & N & $\%$ & $\%$ & $\%$ \\
\hline $\begin{array}{l}\text { Profil Alte Sprachen } \\
\text { Sprachkombination enthält Latein } \\
\text { und/oder Griechisch; Schwerpunkt- } \\
\text { fach ist eine Sprache }\end{array}$ & 285 & 19.9 & $61.5 \%$ & $38.5 \%$ \\
\hline $\begin{array}{l}\text { Profil Neue Sprachen } \\
\text { Sprachkombination enthält nur mo- } \\
\text { derne Sprachen; Schwerpunktfach ist } \\
\text { eine Sprache }\end{array}$ & 485 & 33.8 & $75.9 \%$ & $24.1 \%$ \\
\hline $\begin{array}{l}\text { Profil Mathematik / Natur- } \\
\text { wissenschaften } \\
\text { Schwerpunktfach ist «Biologie und } \\
\begin{array}{l}\text { Chemie» oder «Physik und Anwen- } \\
\text { dungen der Mathematik» }\end{array}\end{array}$ & 202 & 14.1 & $36.3 \%$ & $63.7 \%$ \\
\hline $\begin{array}{l}\text { Profil Musisch } \\
\text { Schwerpunktfach ist «Bildnerisches } \\
\text { Gestalten» oder «Musik» }\end{array}$ & 261 & 18.2 & $83.3 \%$ & $16.7 \%$ \\
\hline $\begin{array}{l}\text { Profil Wirtschaft / Recht } \\
\text { Schwerpunktfach ist «Wirtschaft } \\
\text { und Recht» }\end{array}$ & 186 & 13.0 & $39.9 \%$ & $60.1 \%$ \\
\hline Missing & 1434 & 99.9 & $63.9 \%$ & $36.1 \%$ \\
\hline Total & 1.0 & & \\
\hline
\end{tabular}

Für die vorliegenden Analysen werden 25 Kompetenzindikatoren berücksichtigt, die überfachliche Kompetenzen im personalen, interpersonalen und gesellschaftsbezogenen Bereich erfassen. Die Auswahl erfolgte theoriebezogen und in Abhängigkeit der im MAR formulierten Zielkategorien (Grob \& Maag Merki, 2001; Schweizerischer Bundesrat \& Erziehungsdirektorenkonferenz EDK, 1995). Die teststatistischen Kennwerte wie auch je ein Beispielitem pro Skala sind in Tabelle 2 einsehbar.

Profilspezifische Effekte sowie Interaktionseffekte zwischen den beiden Faktoren «Maturitätsprofil» und "Geschlecht» werden auf der Basis einer 2 x 2 univariaten, multifaktoriellen Kovarianzanalysen untersucht. Als abhängige Variable wird die Kompetenzentwicklung der Jugendlichen vom 10. bis zum 12. Schuljahr berücksichtigt, wobei jeweils die Differenz zwischen dem Kompetenzausmass zum 12. Schuljahr und zum 10. Schuljahr berechnet worden ist.

Als unabhängige Variablen werden pro abhängige Variable die zwei Indikatoren «Geschlecht (junge Frauen/junge Männer)» und «Maturitätsprofil» eingegeben, wobei das jeweilige Maturitätsprofil mit «1», die anderen Profile zusammen mit « 0 » kodiert worden sind. 


\section{Tabelle 2: Kurzcharakterisierung der Konstrukte}

\begin{tabular}{|c|c|c|c|c|}
\hline Konstrukt & Beispielitem & $\begin{array}{l}\text { Anzahl } \\
\text { Items }\end{array}$ & $\begin{array}{l}\text { Cronbachs } \\
\text { Alpha } \\
2001\end{array}$ & $\begin{array}{c}\text { Cronbachs } \\
\text { Alpha } \\
2004\end{array}$ \\
\hline Selbstwert & $\begin{array}{l}\text { Im Grossen und Ganzen bin ich mit mir } \\
\text { zufrieden }\end{array}$ & 6 & .83 & .86 \\
\hline Selbstwirksamkeit & $\begin{array}{l}\text { Wenn ich etwas wirklich will und mir alle } \\
\text { Mühe gebe, kann ich es erreichen }\end{array}$ & 5 & .69 & .74 \\
\hline Selbstreflexion & $\begin{array}{l}\text { Ich nehme mir immer mal wieder Zeit, um } \\
\text { über mich und mein Verhalten nachzudenken }\end{array}$ & 6 & .76 & .79 \\
\hline $\begin{array}{l}\text { Wahrnehmung eige- } \\
\text { ner Gefühle }\end{array}$ & $\begin{array}{l}\text { Ich nehme meine Empfindungen sehr } \\
\text { bewusst wahr }\end{array}$ & 4 & .82 & .83 \\
\hline $\begin{array}{l}\text { Umgang mit belas- } \\
\text { tenden Gefühlen }\end{array}$ & $\begin{array}{l}\text { Von manchen negativen Gedanken kann } \\
\text { ich mich gar nicht mehr loslösen }\end{array}$ & 6 & .80 & .83 \\
\hline Volition & $\begin{array}{l}\text { Wenn ich eine schwierige Aufgabe zu bear- } \\
\text { beiten habe } \\
\text { - schiebe ich sie oft lange Zeit vor mir her } \\
\text { (Inv.) ... }\end{array}$ & 5 & .88 & .91 \\
\hline Planungsstrategien & $\begin{array}{l}\text { Wenn ich eine schwierige Aufgabe zu bear- } \\
\text { beiten habe... } \\
\text { - plane ich genau, wie ich die Aufgabe am } \\
\text { besten lösen kann }\end{array}$ & 5 & .80 & .84 \\
\hline $\begin{array}{l}\text { Transformations- } \\
\text { strategien }\end{array}$ & $\begin{array}{l}\text { Wenn ich eine schwierige Aufgabe zu bear- } \\
\text { beiten habe... } \\
\text { - zeichne ich mir die wichtigsten Dinge auf }\end{array}$ & 4 & .67 & .70 \\
\hline $\begin{array}{l}\text { Elaborations- } \\
\text { strategien }\end{array}$ & $\begin{array}{l}\text { Wenn ich eine schwierige Aufgabe zu bear- } \\
\text { beiten habe... } \\
\text { - erinnere ich mich daran, wie ich } \\
\text { vergleichbare Aufgaben lösen konnte }\end{array}$ & 5 & .70 & .76 \\
\hline Monitoring & $\begin{array}{l}\text { Währenddem ich eine schwierige Aufgabe } \\
\text { bearbeite... } \\
\text { - unterbreche ich manchmal bewusst } \\
\text { meine Arbeit, um sie zu überprüfen }\end{array}$ & 5 & .73 & .79 \\
\hline Persistenz & $\begin{array}{l}\text { Auch wenn ich bei einer Arbeit auf } \\
\text { Schwierigkeiten stosse, bleibe ich } \\
\text { hartnäckig daran }\end{array}$ & 6 & .80 & .83 \\
\hline Evaluationsstrategien & $\begin{array}{l}\text { Nach Abschluss einer schwierigen } \\
\text { Aufgabe... } \\
\text { - versuche ich herauszufinden, was ich } \\
\text { besonders gut und was ich weniger gut } \\
\text { gelöst habe }\end{array}$ & 5 & .72 & .76 \\
\hline $\begin{array}{l}\text { Intrinsische } \\
\text { Motivation }\end{array}$ & $\begin{array}{l}\text { Ich lerne in der Mittelschule... } \\
\text { - weil mir die Arbeit mit den verschiede- } \\
\text { nen Themen in der Schule Spass macht. }\end{array}$ & 4 & .82 & .85 \\
\hline $\begin{array}{l}\text { Extrinsische } \\
\text { Motivation: } \\
\text { materielle Ziele }\end{array}$ & $\begin{array}{l}\text { Ich lerne in der Mittelschule... } \\
\text { - um später ein finanziell abgesichertes } \\
\text { Leben führen zu können. }\end{array}$ & 4 & .87 & .89 \\
\hline
\end{tabular}




\begin{tabular}{|c|c|c|c|c|}
\hline Leistungsmotivation & $\begin{array}{l}\text { Aufgaben, bei denen ich gefordert werde, } \\
\text { machen mir Spass }\end{array}$ & 8 & .78 & .81 \\
\hline Umweltverhalten & $\begin{array}{l}\text { Ich kaufe Produkte, die wirklich umwelt- } \\
\text { schonend verpackt sind (z.B. wiederver- } \\
\text { wertbare Verpackung) }\end{array}$ & 10 & .79 & .78 \\
\hline Umweltwissen & $\begin{array}{l}\text { Was spricht aus der Sicht des Umwelt- } \\
\text { schutzes am meisten dagegen, in der } \\
\text { Schweiz grüne Spargeln aus Kalifornien zu } \\
\text { kaufen? } \\
\text { - Energieverbrauch durch lange Transport- } \\
\text { wege }\end{array}$ & $10 / 15^{*}$ & .73 & .70 \\
\hline $\begin{array}{l}\text { Gesellschaftliche } \\
\text { Verantwortung }\end{array}$ & $\begin{array}{l}\text { Es ist eine wichtige Aufgabe des Staates, } \\
\text { hilfsbedürftige Menschen zu unterstützen. }\end{array}$ & 6 & .72 & 74 \\
\hline $\begin{array}{l}\text { Einstellung zur } \\
\text { Gleichberechtigung } \\
\text { der Geschlechter }\end{array}$ & $\begin{array}{l}\text { Politische Führungspositionen sollten } \\
\text { hauptsächlich Männern gegeben werden }\end{array}$ & 6 & .79 & .79 \\
\hline $\begin{array}{l}\text { Einstellung zu } \\
\text { Migrant/innen }\end{array}$ & $\begin{array}{l}\text { Die in der Schweiz lebenden Ausländer ha- } \\
\text { ben das genau gleiche Recht auf Arbeit wie } \\
\text { Schweizer }\end{array}$ & 4 & .73 & .74 \\
\hline $\begin{array}{l}\text { Politisches und } \\
\text { politökonomisches } \\
\text { Wissen }\end{array}$ & $\begin{array}{l}\text { Was ist der Hauptgrund, warum Kartelle } \\
\text { in der freien Marktwirtschaft ein Problem } \\
\text { darstellen? } \\
\text { - Sie bewirken, dass die Konsumentinnen } \\
\text { und Konsumenten zu hohe Preise bezahlen } \\
\text { müssen }\end{array}$ & $9 / 15^{*}$ & .81 & .82 \\
\hline Politisches Interesse & $\begin{array}{l}\text { Ganz allgemein gefragt: Wie stark interes- } \\
\text { sieren Sie sich für Politik? }\end{array}$ & 1 & - & - \\
\hline Alkoholkonsum & $\begin{array}{l}\text { Wie häufig haben Sie in den letzten } 12 \\
\text { Monaten die folgenden Mittel genommen? } \\
\text { - Alkohol }\end{array}$ & 1 & - & - \\
\hline Tabakkonsum & $\begin{array}{l}\text { Wie häufig haben Sie in den letzten } 12 \\
\text { Monaten die folgenden Mittel genommen? } \\
\text { - Tabak }\end{array}$ & 1 & - & - \\
\hline $\begin{array}{l}\text { Somatische } \\
\text { Beschwerden }\end{array}$ & $\begin{array}{l}\text { Wie häufig leiden Sie an folgenden } \\
\text { Beschwerden? } \\
\text { - Nervosität }\end{array}$ & 6 & .74 & .71 \\
\hline
\end{tabular}

* erste Zahl: Anzahl Items erste Erhebung 2001, zweite Zahl: Anzahl Items zweite Erhebung 2004

Als Kovariate - um mögliche Drittvariableneffekte zu kontrollieren - werden die Variablen «Familiärer Bildungshintergrund» mit den drei Indikatoren «zuletzt abgeschlossene Ausbildung der Mutter bzw. des Vaters» (1=obligatorische Schule, ... 4=Maturität, Lehrerseminar, Universität) und «Anzahl Bücher zuhause» ( $1=0$ bis 10 Bücher, ... 5=über 500) sowie fünf Indikatoren zur Erfassung des Freizeitverhaltens der Jugendlichen eingegeben (zur Bedeutung von Peers siehe z.B. Fend, 1998a; Maag Merki, 2006a; Wigfield, Byrnes \& Eccles, 2006). ${ }^{3}$ Im Fokus stehen Haupteffekte aufgrund der Profilzugehörigkeit und Interaktionseffekte zwischen den Faktoren «Geschlecht» und «Profil» unter Kontrolle 
der Kovariate. Nicht thematisiert werden geschlechtsspezifische Effekte als Haupteffekte.

Zeigen sich hinsichtlich der Kompetenzentwicklung systematische profilspezifische und Interaktionseffekte (Geschlecht x Maturitätsprofil), so kann dies als bedeutsamer Hinweis für einen systematischen Einfluss des Lernkontextes gedeutet werden, da allfällige Selbstselektionseffekte in der Wahl des Maturitätsprofils zu Beginn des 10. Schuljahres kontrolliert sind. Allerdings ist zu berücksichtigen, dass dieses Veränderungsmass nur sensitiv ist hinsichtlich des relativen Niveaus, nicht aber hinsichtlich des absoluten Niveaus der Kompetenzentwicklung der Jugendlichen. Aus diesem Grund werden dieselben Analysen auch für das 10. und 12. Schuljahr durchgeführt. Diese werden allerdings einzig ergänzend im Text und nicht tabellarisch dokumentiert.

Allfällige Mittelwertsdifferenzen im Entwicklungsverlauf werden auf der Basis von Effektgrössen (Cohen, 1988) und in den Tabellen mit Signifikanzniveaus ausgegeben. Effektgrössen ermöglichen es, der über das Signifikanzniveau dokumentierten empirischen Bedeutsamkeit eine inhaltliche Bedeutsamkeit gegenüberzustellen, die die Mittelwertsdifferenz in Abhängigkeit der Heterogenität innerhalb der Gruppe (Standardabweichung) gewichtet. Haupteffekte mit Effektgrössen von $\mathrm{d}<.10$ werden im Text nicht speziell erwähnt, auch wenn sie signifikant sind. Alle dargestellten Effekte weisen höchstens eine Irrtumswahrscheinlichkeit von $\mathrm{p}<.05$ auf.

\section{Ergebnisse}

Werden die Ergebnisse der längsschnittlichen Auswertungen profilübergreifend analysiert, so zeigt sich, dass anzahlmässig die profilspezifischen Effekte (Haupteffekt Profil) die Interaktionseffekte deutlich übertreffen. Die meisten profilspezifischen Effekte zeigen sich in den drei Profilen "musisch», "mathematisch-naturwissenschaftlich» und "Alte Sprachen», die meisten Interaktionseffekte im Profil «Alte Sprachen».

Im musischen Profil, besonders häufig von jungen Frauen gewählt (vgl. Tabelle 1), zeigen sich profilspezifische Effekte bei der Kompetenzentwicklung bis auf eine Ausnahme - beim politischen Wissen - in Bereichen, in denen sich zu Beginn des 10. Schuljahres keinen Haupteffekt Profil ergeben hat (vgl. Tabelle 3). Schüler/innen des musischen Profils haben damit im Vergleich zu ihren Kolleg/innen in den anderen Profilen trotz vergleichbarem Ausgangsniveau signifikant stärker ihre Lernstrategien (Planungsstrategien: $d=.11, \mathrm{p}=.011$; Transformationsstrategien: $\mathrm{d}=.22, \mathrm{p}=.009$; Monitoringstrategien: $\mathrm{d}=.11, \mathrm{p}=.031)$ und ihre Fähigkeit zur Wahrnehmung der eigenen Gefühle $(\mathrm{d}=.16, \mathrm{p}=.046)$ entwickelt. Hingegen zeigt sich ein systematisch geringerer Entwicklungsumfang in Bezug auf das politische Wissen $(\mathrm{d}=-.39, \mathrm{p}=.001)$. Der systematisch geringere Kompetenzzuwachs im politischen Wissen muss zusätzlich in Zusammenhang 
gebracht werden mit dem bereits zu Beginn des 10. Schuljahres deutlich geringeren politischen Wissens der Schüler/innen des musischen Profils im Vergleich zur Gruppe der Schüler/innen der anderen Profile ( $\mathrm{F}=10.891, \mathrm{p}=.001$.), so dass es im Verlaufe des Gymnasiums zu einem Schereneffekt bzw. einer Vergrösserung der Differenz gekommen ist.

Tabelle 3: Univariate, mehrfaktorielle Kovarianzanalyse mit den unabhängigen Variablen "Geschlecht» und "Maturitätsprofil Musisch»; Kovariate: familiärer Bildungshintergrund, Freizeitaktivitäten; $M=$ Musisches Profil, $A=$ Profil Alte Sprachen, N=Profil Neue Sprachen, MN=Profil Mathematik/Naturwissenschaften, WR=Profil Wirtschaft/Recht

\begin{tabular}{|l|l|l|}
\hline & \multicolumn{2}{|l|}{ Entwicklung 10.-12. Schuljahr } \\
\hline Kompetenzindikator & $\begin{array}{l}\text { Unterschied Profil M } \leftrightarrow \\
\text { Profile A, N, MN, WR }\end{array}$ & $\begin{array}{l}\text { Interaktion } \\
\text { Geschlecht x Profil }\end{array}$ \\
\hline Wahrnehmung eigener Gefühle & $\begin{array}{l}\mathrm{F}=3.979, \mathrm{p}=.046 \\
\mathrm{M}>\mathrm{A}, \mathrm{N}, \mathrm{MN}, \mathrm{WR}\end{array}$ & n.s. \\
\hline Planungsstrategien & $\begin{array}{l}\mathrm{F}=6.410, \mathrm{p}=.011 \\
\mathrm{M}>\mathrm{A}, \mathrm{N}, \mathrm{MN}, \mathrm{WR}\end{array}$ & $\mathrm{F}=5.321 \mathrm{p}=.021$ \\
\hline Transformationsstrategien & $\begin{array}{l}\mathrm{F}=6.869, \mathrm{p}=.009 \\
\mathrm{M}>\mathrm{A}, \mathrm{N}, \mathrm{MN}, \mathrm{WR}\end{array}$ & n.s. \\
\hline Monitoringsstrategien & $\begin{array}{l}\mathrm{F}=4.669, \mathrm{p}=.031 \\
\mathrm{M}>\mathrm{A}, \mathrm{N}, \mathrm{MN}, \mathrm{WR}\end{array}$ & n.s. \\
\hline Politisches Wissen & $\begin{array}{l}\mathrm{F}=11.45, \mathrm{p}=.001 \\
\mathrm{M}<\mathrm{A}, \mathrm{N}, \mathrm{MN}, \mathrm{WR}\end{array}$ & n.s. \\
\hline Politisches Interesse & $\begin{array}{l}\mathrm{F}=6.191, \mathrm{p}=.013 \\
\mathrm{M}<\mathrm{A}, \mathrm{N}, \mathrm{MN}, \mathrm{WR}\end{array}$ & F=9.305 $\mathrm{p}=.002$ \\
\hline
\end{tabular}

In zwei Fällen zeigen sich signifikante Interaktionseffekte. Im Entwicklungsverlauf des politischen Interesses vom 10. zum 12. Schuljahr zeigen sich einzig bei den Schülern bedeutsame Unterschiede zwischen den beiden Gruppen (Differenz musisches Profil vs. Restgruppe: $\mathrm{d}=-.53)$, nicht aber bei den Schülerinnen $(\mathrm{d}=.04)$, wobei bei den männlichen Jugendlichen des musischen Profils eine Abnahme des politischen Interesses festgestellt werden kann, während dem bei den Kollegen in der Referenzgruppe wie auch bei den jungen Frauen in beiden Gruppen das politische Interesse zugenommen hat.

Der zweite Interaktionseffekt ergibt sich beim Indikator «Planungsstrategien». Wiederum zeigen sich einzig bei den jungen Männern bedeutsame Unterschiede zwischen den beiden Gruppen $(\mathrm{d}=.47)$, nicht aber bei den Schülerinnen $(\mathrm{d}=.04)$. In diesem Fall ist bei den männlichen Jugendlichen des musischen Profils eine Zunahme des Einsatzes von Planungsstrategien zu beobachten, während dem bei den männlichen Jugendlichen der Restgruppe wie auch bei den jungen Frauen keine systematische Entwicklung vom 10. bis zum 12. Schuljahr sichtbar wird. 
Im Profil «Alte Sprachen», von den jungen Frauen und Männern in etwa entsprechend der Grundgesamtheit gewählt (vgl. Tabelle 1), ergeben sich verschiedene Haupteffekte aufgrund der Profilzugehörigkeit, in den meisten Fällen ohne dass sich ein solcher bereits im 10. Schuljahr gezeigt hätte (vgl. Tabelle 4). Der stärkste Effekt ergibt sich hinsichtlich des politischen Wissens. Die Schüler/innen des altsprachlichen Profils weisen eine deutlich stärkere Kompetenzentwicklung auf als die Schüler/innen der anderen Profile $(\mathrm{d}=.45$, $\mathrm{p}=.000)$. Zusätzlich zum Haupteffekt wird auch ein Interaktionseffekt sichtbar, da die geschlechtsspezifischen Unterschiede im Entwicklungsverlauf, wie sie sich in der Gruppe der anderen Profil zeigen, bei den Schüler/innen des altsprachlichen Profils nicht sichtbar werden. Damit unterscheiden sich die weiblichen Jugendlichen dieses Profils ( $\mathrm{d}=.64)$ bedeutend stärker von ihren Kolleginnen in der Referenzgruppe als die männlichen Jugendlichen $(\mathrm{d}=.16)$.

Tabelle 4: Univariate, mehrfaktorielle Kovarianzanalyse mit den unabhängigen Variablen «Geschlecht» und «Maturitätsprofil Alte Sprachen»; Kovariate: familiärer Bildungshintergrund, Freizeitaktivitäten; $M=$ Musisches Profil, $A=$ Profil Alte Sprachen, N=Profil Neue Sprachen, MN=Profil Mathematik/Naturwissenschaften, WR=Profil Wirtschaft/Recht

\begin{tabular}{|l|l|l|}
\hline & \multicolumn{2}{|l|}{ Entwicklung 10.-12. Schuljahr } \\
\hline Kompetenzindikator & $\begin{array}{l}\text { Unterschied Profil A } \leftrightarrow \\
\text { Profile WR, M, N, MN }\end{array}$ & $\begin{array}{l}\text { Interaktion } \\
\text { Geschlecht x Profil }\end{array}$ \\
\hline Alkoholkonsum & $\begin{array}{l}\mathrm{F}=8.006, \mathrm{p}=.003 \\
\mathrm{~A}>\mathrm{WR}, \mathrm{M}, \mathrm{N}, \mathrm{MN}\end{array}$ & n.s. \\
\hline $\begin{array}{l}\text { Einstellung gegenüber der Gleich- } \\
\text { berechtigung der Geschlechter }\end{array}$ & $\begin{array}{l}\mathrm{F}=4.427, \mathrm{p}=.036 \\
\mathrm{~A}<\mathrm{WR}, \mathrm{M}, \mathrm{N}, \mathrm{MN}\end{array}$ & $\mathrm{F}=5.321 \mathrm{p}=.021$ \\
\hline Leistungsmotivation & $\begin{array}{l}\mathrm{F}=4.651, \mathrm{p}=.031 \\
\mathrm{~A}>\mathrm{WR}, \mathrm{M}, \mathrm{N}, \mathrm{MN}\end{array}$ & $\mathrm{F}=9.985, \mathrm{p}=.002$ \\
\hline Selbstwert & n.s. & $\mathrm{F}=5.523, \mathrm{p}=.019$ \\
\hline $\begin{array}{l}\text { Umgang mit belastenden } \\
\text { Gefühlen }\end{array}$ & $\begin{array}{l}\mathrm{F}=5.717, \mathrm{p}=.017 \\
\mathrm{~A}>\mathrm{WR}, \mathrm{M}, \mathrm{N}, \mathrm{MN}\end{array}$ & n.s. \\
\hline Elaborationsstrategien & $\begin{array}{l}\mathrm{F}=5.423, \mathrm{p}=.020 \\
\mathrm{~A}>\mathrm{WR}, \mathrm{M}, \mathrm{N}, \mathrm{MN}\end{array}$ & $\mathrm{F}=9.305 \mathrm{p}=.002$ \\
\hline Evaluationsstrategien & $\begin{array}{l}\mathrm{F}=4.498, \mathrm{p}=.034 \\
\mathrm{~A}>\mathrm{WR}, \mathrm{M}, \mathrm{N}, \mathrm{MN}\end{array}$ & n.s. \\
\hline Politisches Wissen & $\begin{array}{l}\mathrm{F}=27.065, \mathrm{p}=.000 \\
\mathrm{~A}>\mathrm{WR}, \mathrm{M}, \mathrm{N}, \mathrm{MN}\end{array}$ & $\mathrm{F}=9.021, \mathrm{p}=.003$ \\
\hline Politisches Interesse & $\begin{array}{l}\mathrm{F}=3.854, \mathrm{p}=.050 \\
\mathrm{~A}>\mathrm{WR}, \mathrm{M}, \mathrm{N}, \mathrm{MN}\end{array}$ & $\mathrm{F}=4.395, \mathrm{p}=.036$ \\
\hline
\end{tabular}

Bei der Entwicklung des politischen Interesses ist der Haupteffekt zugunsten der Schüler/innen des altsprachlichen Profils bedeutend kleiner $(\mathrm{d}=.10, \mathrm{p}=.050)$. 
Der signifikante Interaktionseffekt bezieht sich auf systematische Unterschiede zwischen den Schülern des altsprachlichen Profils und der anderen Profile $(\mathrm{d}=.26)$, der sich bei den Schülerinnen nicht zeigt $(\mathrm{d}=.01)$, wobei die Schüler des altsprachlichen Profils in einem stärkeren Ausmass ihr politisches Interesse entwickelt haben als ihre Kollegen in den anderen Profilen.

Bedeutsame Haupteffekte ergeben sich in weiteren Bereichen. So zeichnen sich die Schüler/innen des altsprachlichen Profils durch eine ausgeprägtere Entwicklung der Leistungsmotivation ( $\mathrm{d}=.11$, $\mathrm{p}=.031)$, der Fähigkeit, mit belastenden Gefühlen umzugehen $(\mathrm{d}=.16, \mathrm{p}=.017)$ sowie des Einsatzes von Elaborations$(\mathrm{d}=.16, \mathrm{p}=.020)$ und Evaluationsstrategien $(\mathrm{d}=.17, \mathrm{p}=.034)$ aus. Bei der Leistungsmotivation ergibt sich zusätzlich zum Haupteffekt auch ein Interaktionseffekt. Zeigt sich im Entwicklungsverlauf bei den Schülerinnen des altsprachlichen Profils nur ein geringer Unterschied zu den Schülerinnen der anderen Profile $(\mathrm{d}=-.08)$, ist dieser Unterschied bei den Schülern im altsprachlichen Profil im Vergleich zu ihren Kollegen deutlich stärker $(\mathrm{d}=.39)$. Die Schüler des altsprachlichen Profils haben damit in stärkerem Ausmass ihre Leistungsmotivation entwickelt als ihre Kollegen in den anderen Profilen und die weiblichen Jugendlichen.

In zwei Fällen zeigt sich zusätzlich zum Haupteffekt zu Beginn des 10. Schuljahres ebenfalls ein Haupteffekt im Entwicklungsverlauf. Während dem die Schüler/innen zu Beginn des 10. Schuljahres einen systematisch geringeren Alkoholkonsum aufgewiesen haben $(\mathrm{F}=15.570, \mathrm{p}=.000)$, ergibt sich im altsprachlichen Profil eine stärkere Zunahme des Konsums als in der Referenzgruppe $(\mathrm{d}=.22, \mathrm{p}=.003)$. Der stärkere Zuwachs im Alkoholkonsum führt damit zu einer Angleichung des Alkoholkonsums bei den Schüler/innen dieses Profils bis zum Ende des Gymnasiums. Der Befund hinsichtlich der Einstellung gegenüber der Gleichberechtigung der Geschlechter ist gerade gegenläufig. Während dem die Schüler/innen des altsprachlichen Profils zu Beginn des 10. Schuljahres eine signifikant positivere Einstellung kommuniziert haben $(\mathrm{F}=4.988, \mathrm{p}=.026)$, zeigt sich im Entwicklungsverlauf eine geringere Ausprägung $(\mathrm{d}=-.13, \mathrm{p}=.036)$, wobei sich am Ende des Gymnasiums die Differenz zwischen diesem Profil und den anderen Profilen ebenfalls angeglichen hat.

Ein letzter Interaktionseffekt ist in der Entwicklung des Selbstwertes zu beobachten, ohne dass sich diesbezüglich auch ein Haupteffekt im 10. Schuljahr oder im Entwicklungsverlauf ergeben hätte. Analog zum Ergebnis der Entwicklung der Leistungsmotivation zeigt sich im Entwicklungsverlauf bei den Schülerinnen des altsprachlichen Profils nur ein geringer Unterschied zu den Schülerinnen der anderen Profile (d=-.10). Hingegen fällt dieser Unterschied bei den Schülern im altsprachlichen Profil im Vergleich zu ihren Kollegen deutlich stärker aus $(\mathrm{d}=.25)$. Die Selbstwertentwicklung verläuft damit bei den Schülern des altsprachlichen Profils besonders prägnant.

Im mathematisch-naturwissenschaftlichen Profil, besonders häufig von den jungen Männern gewählt (vgl. Tabelle 1), zeigen sich ebenfalls verschiedene 
Haupteffekte, hingegen keine Interaktionseffekte (vgl. Tabelle 5). Die Entwicklungen verlaufen damit für beide Geschlechter analog. Der stärkste Haupteffekt zeigt sich in der Entwicklung des politischen Wissens, welche bei den Jugendlichen dieses Profils ausgeprägter verläuft als bei den Jugendlichen der restlichen Profile $(\mathrm{d}=.33, \mathrm{p}=.008)$, obwohl die Schüler/innen dieses Profils bereits zu Beginn des 10. Schuljahres ein signifikant ausgeprägteres Wissen aufgewiesen haben $(\mathrm{F}=4.933, \mathrm{p}=.027)$.

Tabelle 5: Univariate, mehrfaktorielle Kovarianzanalyse mit den unabhängigen Variablen "Geschlecht» und "Maturitätsprofil Mathematik/Naturwissenschaften»; Kovariate: familiärer Bildungshintergrund, Freizeitaktivitäten; $M=$ Musisches Profil, A=Profil Alte Sprachen, N=Profil Neue Sprachen, MN=Profil Mathematik/Naturwissenschaften, WR=Profil Wirtschaft/Recht

\begin{tabular}{|l|l|l|}
\hline & \multicolumn{2}{|l|}{ Entwicklung 10.-12. Schuljahr } \\
\hline Kompetenzindikator & $\begin{array}{l}\text { Unterschied Profil MN } \\
\text { Profile A, M, M, WR }\end{array}$ & $\begin{array}{l}\text { Interaktion } \\
\text { Geschlecht x Profil }\end{array}$ \\
\hline Selbstwert & $\begin{array}{l}\mathrm{F}=9.057, \mathrm{p}=.003 \\
\mathrm{MN}<\text { Profile A, M, N, WR }\end{array}$ & n.s. \\
\hline Selbstwirksamkeit & $\begin{array}{l}\mathrm{F}=4.313, \mathrm{p}=.038 \\
\mathrm{MN}<\text { Profile A, M, N, WR }\end{array}$ & n.s. \\
\hline Elaborationsstrategien & $\begin{array}{l}\mathrm{F}=4.208, \mathrm{p}=.040 \\
\mathrm{MN}<\text { Profile A, M, N, WR }\end{array}$ & n.s. \\
\hline Transformationsstrategien & $\begin{array}{l}\mathrm{F}=3.850, \mathrm{p}=.050 \\
\mathrm{MN}<\text { Profile A, M, N, WR }\end{array}$ & n.s. \\
\hline Politisches Wissen & $\begin{array}{l}\mathrm{F}=7.001, \mathrm{p}=.008 \\
\mathrm{MN}>\text { Profile A, M, N, WR }\end{array}$ & n.s. \\
\hline Umweltverhalten & $\begin{array}{l}\mathrm{F}=3.915, \mathrm{p}=.048 \\
\mathrm{MN}<\text { Profile A, M, N, WR }\end{array}$ & n.s. \\
\hline
\end{tabular}

In den anderen Bereichen zeigen sich etwas moderatere Entwicklungsverläufe der Jugendlichen des mathematisch-naturwissenschaftlichen Profils im Vergleich zur Referenzgruppe, so hinsichtlich der Entwicklung der Selbstwirksamkeitserwartungen $(\mathrm{d}=-.14, \mathrm{p}=.038)$, des Einsatzes von Elaborations- $(\mathrm{d}=-.14, \mathrm{p}=.040)$ und Transformationsstrategien $(\mathrm{d}=-.15, \mathrm{p}=.050)$ und des umweltbewussten Verhaltens $(\mathrm{d}=-.13, \mathrm{p}=.048)$. Diese profilspezifischen Effekte zeigen sich einzig im Entwicklungsverlauf, nicht aber bereits zum Beginn des 10. Schuljahres. Hinsichtlich der Entwicklung des Selbstwertes ergibt sich auch ein signifikanter Haupteffekt, indem die Schüler/innen des mathematisch-naturwissenschaftlichen Profils ihren Selbstwert in geringerem Ausmass entwickelt haben als die Schüler/innen der Referenzgruppe $(\mathrm{d}=-.23, \mathrm{p}=.003)$. Allerdings weisen die Schüler/innen dieses Profils zu Beginn des 10. Schuljahres einen signifikant höheren Selbstwert als ihre Referenzgruppe auf $(\mathrm{F}=4.635, \mathrm{p}=.032)$, so dass sich die Diffe- 
renzen bis zum Ende des Gymnasiums zwischen den beiden Gruppen angeglichen haben und am Ende des Gymnasiums keine signifikanten Unterschiede mehr bestehen.

Im Gegensatz zu den bereits beschriebenen Profilen gibt es im Profil "Neue Sprachen», besonders häufig von weiblichen Jugendlichen gewählt (vgl. Tabelle 1), nur einen substanziellen Haupteffekt (vgl. Tabelle 6). So entwickelten die Schüler/innen dieses Profils in bedeutend geringerem Masse als die Schüler/innen der restlichen Profile ihr politisches Wissen $(\mathrm{d}=-.31, \mathrm{p}=.017)$. Dieser Unterschied zwischen den beiden Gruppen ist im 10. Schuljahr noch nicht sichtbar gewesen. Zusätzlich zeigt sich hinsichtlich der Entwicklung des politischen Wissens ein signifikanter Interaktionseffekt. Der beschriebene Unterschied zwischen diesen beiden Gruppen ist einzig bei den weiblichen Jugendlichen bedeutsam $(\mathrm{d}=-.35)$, nicht aber bei den männlichen $(\mathrm{d}=.05)$. So hat sich bei den Schülerinnen des neusprachlichen Profils das politische Wissen in bedeutend geringerem Ausmass entwickelt als bei den Schülerinnen der restlichen Profile.

Tabelle 6: Univariate, mehrfaktorielle Kovarianzanalyse mit den unabhängigen Variablen "Geschlecht» und "Maturitätsprofil Neue Sprachen»; Kovariate: familiärer Bildungshinter-grund, Freizeitaktivitäten; $M=$ Musisches Profil, A=Profil Alte Sprachen, N=Profil Neue Sprachen, MN=Profil Mathematik/Naturwissenschaften, WR=Profil Wirtschaft/Recht

\begin{tabular}{|l|l|l|}
\hline & \multicolumn{2}{|l|}{ Entwicklung 10.-12. Schuljahr } \\
\hline Kompetenzindikator & $\begin{array}{l}\text { Unterschied Profil N } \\
\text { Profile A, M, MN, WR }\end{array}$ & $\begin{array}{l}\text { Interaktion } \\
\text { Geschlecht } x \text { Profil }\end{array}$ \\
\hline $\begin{array}{l}\text { Extrinsische Motivation - } \\
\text { materielle Ziele }\end{array}$ & $\begin{array}{l}\mathrm{F}=3.907, \mathrm{p}=.048 \\
\mathrm{~N}>\mathrm{A}, \mathrm{M}, \mathrm{MN}, \mathrm{WR}\end{array}$ & $\mathrm{F}=8.228, \mathrm{p}=.004$ \\
\hline Politisches Wissen & $\begin{array}{l}\mathrm{F}=5.746, \mathrm{p}=.017 \\
\mathrm{~N}<\mathrm{A}, \mathrm{M}, \mathrm{MN}, \mathrm{WR}\end{array}$ & $\mathrm{F}=4.802, \mathrm{p}=.029$ \\
\hline
\end{tabular}

Ein weiterer bedeutsamer Interaktionseffekt zeigt sich in Bezug auf die Bedeutung von materiellen Zielen beim Lernen. Hier ergeben sich bedeutsame Unterschiede zwischen den beiden Gruppen einzig bei den männlichen $(\mathrm{d}=.35)$, nicht aber bei den weiblichen Jugendlichen $(\mathrm{d}=.05)$, wobei die Schüler des neusprachlichen Profils in weitaus stärkerem Masse ihre extrinsische Lernmotivation entwickelt haben als die Schüler der restlichen Profile. Für die Interpretation dieses Befundes ist wichtig zu wissen, dass die Schüler des neusprachlichen Profils zu Beginn des 10. Schuljahres in deutlich geringerem Ausmass materielle Ziele als relevant kommuniziert haben als ihre Kollegen der anderen Profile, ein Effekt, der sich bei den jungen Frauen nicht gezeigt hat $(\mathrm{F}=14.038, \mathrm{p}=.000)$. Am Ende des 12. Schuljahres zeigen sich in der Folge keine signifikanten Effekte mehr zwischen den Gruppen. 
Im Profil "Wirtschaft/Recht», besonders häufig von männlichen Jugendlichen gewählt (vgl. Tabelle 1), lassen sich drei Haupteffekte und zwei Interaktionseffekte identifizieren (vgl. Tabelle 7), allesamt in Bereichen, in denen sich bereits zu Beginn des 10. Schuljahres substanzielle Differenzen gezeigt haben. Zeichneten sich die Schüler/innen dieses Profil im 10. Schuljahr durch eine substanziell negativere Einstellung gegenüber Migrant/innen $(\mathrm{F}=27.248, \mathrm{p}=.000)$ und der Gleichberechtigung der Geschlechter aus ( $\mathrm{F}=9.741, \mathrm{p}=.002)$, so ist in beiden Bereichen bis zum Ende des Gymnasiums eine positivere Entwicklung als bei den Schüler/innen der anderen Profile erkennbar (Einstellung gegenüber Migrant/innen: $\mathrm{d}=.27, \mathrm{p}=.011$; Gleichberechtigung der Geschlechter: $\mathrm{d}=.35, \mathrm{p}=.011$ ). Der bedeutsame Interaktionseffekt bei der Einstellung zur Gleichberechtigung der Geschlechter, der in der signifikanten Differenz im Entwicklungsverlauf bei den männlichen Jugendlichen des Profils «Wirtschaft/Recht» zu den männlichen Jugendlichen in den Restprofilen begründet ist $(\mathrm{d}=.31)$, ohne dass sich ein solcher bei den jungen Frauen ebenfalls gezeigt hätte $(\mathrm{d}=.06)$, führt dazu, dass die im 10. Schuljahr identifizierten geschlechts- und profilspezifischen Differenzen am Ende des Gymnasiums nicht mehr signifikant sind. Bei der Einstellung zu Migrant/innen lässt sich allerdings auch am Ende des Gymnasiums eine signifikante Differenz zu ungunsten der Schüler/innen des Profils "Wirtschaft/Recht» feststellen ( $\mathrm{F}=6.857, \mathrm{p}=.009)$.

Tabelle 7: Univariate, mehrfaktorielle Kovarianzanalyse mit den unabhängigen Variablen "Geschlecht» und "Maturitätsprofil Wirtschaft/Recht»; Kovariate: familiärer Bildungshinter-grund, Freizeitaktivitäten; $M=$ Musisches Profil, $A=$ Profil Alte Sprachen, $N=$ Profil Neue Sprachen, $M N=$ Profil Mathematik/Naturwissenschaften, WR=Profil Wirtschaft/Recht

\begin{tabular}{|l|l|l|}
\hline & \multicolumn{2}{|l|}{ Entwicklung 10.-12. Schuljahr } \\
\hline Kompetenzindikator & $\begin{array}{l}\text { Unterschied Profil WR } \leftrightarrow \\
\text { Profile A, M, N, MN }\end{array}$ & $\begin{array}{l}\text { Interaktion } \\
\text { Geschlecht x Profil }\end{array}$ \\
\hline Einstellung zu Migrant/innen & $\begin{array}{l}\mathrm{F}=6.435, \mathrm{p}=.011 \\
\mathrm{WR}>\mathrm{A}, \mathrm{M}, \mathrm{N}, \mathrm{MN}\end{array}$ & n.s. \\
\hline $\begin{array}{l}\text { Einstellung zur Gleichberech- } \\
\text { ti-gung der Geschlechter }\end{array}$ & $\begin{array}{l}\mathrm{F}=6.542, \mathrm{p}=.011 \\
\text { WR }>\mathrm{A}, \mathrm{M}, \mathrm{N}, \mathrm{MN}\end{array}$ & $\mathrm{F}=3.949, \mathrm{p}=.047$ \\
\hline Politisches Wissen & n.s. & $\mathrm{F}=11.116, \mathrm{p}=.001$ \\
\hline Alkoholkonsum & $\begin{array}{l}\mathrm{F}=8.939, \mathrm{p}=.003 \\
\text { WR }<\mathrm{A}, \mathrm{M}, \mathrm{N}, \mathrm{MN}\end{array}$ & n.s. \\
\hline
\end{tabular}

Ein dritter Haupteffekt im Entwicklungsverlauf ergibt sich hinsichtlich des Alkoholkonsums. Zeichneten sich die Schüler/innen zu Beginn des 10. Schuljahres durch einen stärkeren Alkoholkonsum aus $(\mathrm{F}=33.650, \mathrm{p}=.000)$, so ergibt sich eine signifikante Differenz in der Zunahme des Alkoholkonsums bis zum Ende 
des Gymnasiums im Vergleich zu den Schüler/innen der anderen Profile ( $\mathrm{d}=-.24$, $\mathrm{p}=.003$ ), wobei diese bei den Schüler/innen des Profils «Wirtschaft/Recht» weniger stark ausgefallen ist. Allerdings ist zu berücksichtigen, dass trotz dieses Effektes die Schüler/innen des Profils «Wirtschaft/Recht» am Ende des Gymnasiums einen höheren Alkoholkonsum aufweisen als die Schüler/innen der anderen Profile $(\mathrm{F}=8.215, \mathrm{p}=.004)$.

Ein letzter bedeutsamer Interaktionseffekt zeigt sich in der Entwicklung des politischen Wissens. Die Differenz zwischen dem Profil «Wirtschaft/Recht» und den anderen Profilen fällt bei den jungen Frauen zugunsten des Profils «Wirtschaft/Recht» ( $\mathrm{d}=.37)$, bei den jungen Männern zugunsten der Gruppe der anderen Profile aus $(\mathrm{d}=-.20)$.

\section{Diskussion}

In diesem Beitrag wurde der Frage nachgegangen, inwiefern in der gymnasialen Ausbildung auf der Sekundarstufe II in der Entwicklung von überfachlichen Kompetenzen (Grob \& Maag Merki, 2001) profilspezifische sowie geschlechtsspezifische Effekte in Abhängigkeit des Lernkontextes, d.h. des gewählten Maturitätsprofils, sichtbar werden. Es wurde davon ausgegangen, dass Maturitätsprofile als Institutionalisierung schulischer Ausbildungen in einem bedeutsamen Zusammenhang stehen mit der Entwicklung überfachlicher Kompetenzen der Schüler/innen. Analog zu den schultypenspezifischen Unterschieden in den fachlichen Leistungen (Watermann, Nagy \& Köller, 2004) findet diese These auch im Bereich der überfachlichen Kompetenzen Bestätigung (These 1). Die Ergebnisse verweisen darauf, dass die Entwicklungsverläufe in den letzten drei Schuljahren im Gymnasium in Abhängigkeit des Ausgangsniveaus im 10. Schuljahr sowohl profil- wie auch geschlechtsspezifisch geprägt sind, wobei nicht in jedem Profil gleichermassen solche Effekte auftreten.

In der Mehrheit verlaufen diese Entwicklungen für junge Männer und Frauen analog, indem sich einzig ein Haupteffekt «Maturitätsprofil», nicht aber Interaktionseffekte «Maturitätsprofil x Geschlecht» gezeigt haben. Es können aber spezifische Kompetenzdimensionen identifiziert werden, in denen Maturitätsprofile einen je eigene Entwicklungs- und Sozialisationskontext darstellen, der dazu führt, dass sich die jungen Männer bzw. jungen Frauen zunehmend stärker von ihren Alterskollegen bzw. Alterskolleginnen in den anderen Profilen unterscheiden (These 2), wobei die männlichen Jugendlichen entsprechend den Erwartungen häufiger von diesem differenziellen Entwicklungsmilieu profitieren als die weiblichen Jugendlichen (These 2a). Die profilübergreifenden Vergleiche zeigen aber, dass das numerische Verhältnis zwischen den Geschlechtern in den einzelnen Profilen diesbezüglich kein erklärungsmächtiger Faktor ist (These 2b). 


\section{Diskussion der Haupteffekte «Maturitätsprofil»}

Haupteffekte sind in allen fünf Maturitätsprofilen zu finden, wobei sich diese entsprechend den Erwartungen vor allem bei den Lernkompetenzen und gesellschaftsbezogenen Kompetenzen, nur in einzelnen Fällen aber bei den personalen Kompetenzen zeigen. Die unterschiedliche Plastizität von überfachlichen Kompetenzen in Abhängigkeit der Maturitätsprofile könnte mit der relativen Stabilität auf individueller Ebene, die bei einzelnen personalen Kompetenzen grösser ist als beispielsweise bei Lernkompetenzen (Maag Merki, 2006a), in einem Zusammenhang stehen.

Bei den Schüler/innen des altsprachlichen Profils können am häufigsten profilspezifische Effekte im Entwicklungsverlauf beobachtet werden, ohne dass sich solche bereits zu Beginn des 10. Schuljahres gezeigt haben. Die profilspezifischen Effekte ergeben sich insbesondere im Lernbereich (Leistungsmotivation, Lernstrategien), aber auch im personalen (Umgang mit belastenden Gefühlen) sowie im politischen Bereich, wobei die Schüler/innen in den allermeisten Bereichen Kompetenzen zugewonnen haben. Die Schüler/innen in diesem Profil finden damit einen Lernkontext vor, welcher besonders funktional für eine produktive Kompetenzentwicklung ist und dazu führt, dass sich am Ende des Gymnasiums diese Schüler/innen deutlich von den Schüler/innen der anderen Profile systematisch unterscheiden (Maag Merki, 2006b, S. 183).

Die beim altsprachlichen Profil festgestellten Entwicklungen lassen sich als Ausdifferenzierungen des Kompetenzprofils der Schüler/innen dieses Profils im Vergleich zu den anderen Profilen beschreiben. Dieses Muster der Entwicklungen (Ausdifferenzierung I) findet sich, bis auf das Profil «Wirtschaft/Recht», in allen Profilen: Es ist charakterisiert durch keine profilspezifischen Effekte zu Beginn des 10. Schuljahres sowie profilspezifische Effekt im Entwicklungsverlauf. Besonders häufig können diese im musischen, mathematisch-naturwissenschaftlichen und altsprachlichen Profil identifiziert werden. Profilspezifische Differenzen im Entwicklungsverlauf lassen sich damit weniger auf über die Wahl des Maturitätsprofils ausgelöste Selbstselektionseffekte zu Beginn des 10. Schuljahres, sondern auf schulspezifische Faktoren zurückführen. Aufgrund der vorliegenden Ergebnisse kann nicht bestimmt werden, welche Faktoren mit welchem Gewicht diese Entwicklungen beeinflusst haben. Aufgrund der multifaktoriellen Bestimmtheit von Kompetenzentwicklungen (Helmke \& Weinert, 1997) ist aber nicht davon auszugehen, dass einzig ein einzelner Faktor (z.B. Anzahl Schuljahresstunden in einem Fach) die Varianz in den Kompetenzen der Schüler/innen genügend erklären kann. Zu untersuchen ist daher, durch welche Faktorenkonstellationen sich die einzelnen Profile auszeichnen und mit welchem Gewicht unterrichtsbezogene und formale Faktoren die Entwicklungen beeinflusst haben.

Dies ist auch der Fall beim nachfolgenden Ergebnismuster «Ausdifferenzierung II». So lässt sich diese Variante des ersten Entwicklungsmusters dadurch charakterisieren, dass sich zwar bereits zum Zeitpunkt des 10. Schuljahres profilspezifische Unterschiede gezeigt haben. Allerdings haben sich diese differen- 
ziellen Effekte in den nachfolgenden Schuljahren zusätzlich noch signifikant verstärkt. Dieses Muster lässt sich nur in zwei Fällen finden. Die Schüler/innen des musischen Profils hatten bereits zu Beginn des 10. Schuljahres über ein bedeutend geringeres politisches Wissen verfügt als ihre Kolleg/innen der anderen Profile. Im Verlauf der nachfolgenden drei Schuljahre hat sich dieser Befund noch verschärft. Gerade gegenteilig ist das Ergebnisbild bei den Schüler/innen des mathematisch-naturwissenschaftlichen Profils. Diese verfügten bereits im 10. Schuljahr über ein ausgeprägteres politisches Wissen, und diese Differenz hat sich im Verlaufe der Schuljahre noch vergrössert. Zu vermuten ist, dass diese Ergebnisse aufgrund der ungleichen Verteilung der Geschlechter in diesen beiden Profilen (vgl. Tabelle 1) mit dem auf individueller Ebene immer wieder bestätigten Befund, dass Frauen an politischen und wirtschaftlichen Fragen weniger interessiert sind und geringere Kenntnisse aufweisen als Männer (Kersten, 2003; Sandmeier \& Maag Merki, 2006; Schmid, 2003), sowie mit den spezifischen profilspezifischen Ausbildungskonzepten in einem Zusammenhang stehen. Der für diese Analysen wichtigere zweite Einflussbereich ist in zukünftigen Studien nicht nur hinsichtlich des offiziellen Curriculums in den verschiedenen Profilen zu untersuchen, sondern auch, inwiefern interaktive Prozesse zwischen Lehrpersonen und Schüler/innen bei Themen wie Politik oder Wirtschaft im Sinne des «heimlichen Lehrplanes» (Zinnecker, 1975) vorzufinden sind.

Ein weiteres Muster der Kompetenzentwicklung kann als «Angleichung» bezeichnet werden. Es findet sich einzig bei den Schüler/innen des altsprachlichen Profils und des Profils «Wirtschaft/Recht» und lässt sich dadurch kennzeichnen, dass sich die im 10. Schuljahr vorzufindenden profilspezifisch ausgeprägten Kompetenzdifferenzen im Verlauf der drei Schuljahre reduziert haben. Im altsprachlichen Profil führt dies dazu, dass die im 10. Schuljahr festgestellten Differenzen (geringerer Alkoholkonsum, positivere Einstellung gegenüber der Gleichberechtigung der Geschlechter) am Ende des Gymnasiums nicht mehr signifikant sind. Aus methodischer Perspektive kann hier allerdings vermutet werden, dass es sich dabei um einen Deckeneffekt handeln könnte. Insbesondere bei der Einstellung gegenüber der Gleichberechtigung der Geschlechter haben die Schüler/innen des altsprachlichen Profils zu Beginn des 10. Schuljahres bereits ein sehr positives Bild gezeichnet. Auf eine eher inhaltliche Begründung verweisen die Befunde im Profil «Wirtschaft/Recht». Bei diesen Ergebnissen lässt sich am Ende des Gymnasiums aufgrund der differenziellen Entwicklungsverläufe in zwei Dimensionen (negativere Einstellung zu Migrant/innen, stärkerer Alkoholkonsum) eine Angleichung, nicht aber eine Aufhebung der Kompetenzdifferenzen feststellen.

Das dritte Entwicklungsmuster ist als «Stabilität» zu bezeichnen und verweist auf den Befund, dass die im 10. Schuljahr festgestellten Differenzen oder NichtDifferenzen aufgrund der nicht vorzufindenden profilspezifischen Effekte im Entwicklungsverlauf bestehen bleiben. Dieses Entwicklungsmuster kommt in allen Profilen anzahlmässig am häufigsten vor und deutet darauf hin, dass die 
Kompetenzentwicklung in einzelnen Bereichen nicht durch profilspezifische Effekte beeinflusst wird.

Insgesamt kann vermutet werden, dass in jenen Bereichen, in denen es zwischen den Schüler/innen bereits zu Beginn des 10. Schuljahres systematische profilspezifische Unterschiede in den erfassten überfachlichen Kompetenzen gegeben hat, nur ein geringer Einfluss des Maturitätsprofils auf den Entwicklungs-verlauf sichtbar wird. Dies zeigt sich in der Dominanz des Entwicklungsmusters "Stabilität» sowie im nur geringen Auftreten der Entwicklungsmuster "Angleichung» und «Ausdifferenzierung II». Zu vermuten ist, dass dies auf die relative Stabilität verschiedener überfachlicher Kompetenzdimensionen zurückzuführen ist, die bewirkt, dass der schulische Einfluss auf die Kompetenzentwicklung nur eingeschränkt möglich ist (Maag Merki, 2006a). Zudem kann vermutet werden, dass die schulischen Faktoren auf aggregierter Ebene des Maturitätsprofils für die Kompetenzentwicklung im überfachlichen Bereich weniger einflussstark sind wie die schulischen Faktoren auf individueller Ebene (Eder, 1996; Rakoczy, 2005). Aufgrund der profilspezifischen Unterschiede hinsichtlich der Auftretenshäufigkeit und -stärke der beobachteten Effekte kann des Weiteren vermutet werden, dass die Maturitätsprofile nicht insgesamt für die Kompetenzentwicklung effektvoll sind, sondern unter differenzieller Perspektive beleuchtet werden müssen. So scheint das musische, altsprachliche und mathematisch-naturwissenschaftliche Profil in einem stärkeren Ausmass einen Einfluss auf den Entwicklungsverlauf der Schüler/innen zu haben. Geringer ist der Einfluss im neusprachlichen Profil und im Profil «Wirtschaft/Recht». Für das Verstehen dieser Unterschiede könnte das von Fend (1998b) beschriebene «Angebots-Nutzungsmodell» schulischer Qualität von zentraler Bedeutung sein. Maturitätsprofile werden dann effektvoll für die Kompetenzentwicklung der Schüler/innen, wenn das von schulischer Seite bereitgestellte Angebot optimal auf die Nutzungsmöglichkeiten der Schüler/innen abgestimmt ist. Weniger zentral dürfte die Tatsache sein, wie häufig diese Profile von den beiden Geschlechtern gewählt werden, finden sich doch in beiden Gruppen Profile mit einer Überrepräsentation von jungen Frauen bzw. einer Überrepräsentation von jungen Männern.

\section{Diskussion der Interaktionseffekte "Maturitätsprofil $x$ Geschlecht»}

Auch wenn anzahlmässig nur wenige Interaktionseffekte sichtbar werden, die Entwicklungen für die beiden Geschlechter damit mehrheitlich analog verlaufen, so lässt sich doch sagen, dass in spezifischen Kompetenzdimensionen Maturitätsprofile für die jungen Männer und Frauen je eigene Entwicklungsmilieus darstellen und somit einen differenziellen Effekt erzeugen. Die Ergebnisse dieser Analysen zeigen nun entsprechend den Erwartungen (Heintz et al. 1997), dass dieser Effekt vor allem bei den jungen Männern und weniger bei den jungen Frauen zu beobachten ist. 
Wie bei den profilspezifischen Haupteffekten zeigen sich die meisten Interaktionseffekte im altsprachlichen Profil, einem Profil, das von den männlichen und weiblichen Jugendlichen entsprechend der Grundgesamtheit gewählt wird und damit in Anlehnung an die Begrifflichkeiten in der Arbeitswelt (Heintz et al, 1997) nicht als "go-between-Profil» bezeichnet werden kann. In allen vier Bereichen (Leistungsmotivation, Selbstwert, Politisches Wissen, Politisches Interesse) unterscheiden sich die jungen Männer im Entwicklungsverlauf systematisch von ihren Kollegen, haben somit, trotz vergleichbarem Ausgangsniveau im 10. Schuljahr im stärkeren Ausmass ihre Leistungsmotivation, ihre Selbstwertschätzung und ihre politischen Kompetenzen entwickelt als ihre Kollegen der anderen Profile. Weitere Interaktionseffekte - ein Effekt im Profil «Neue Sprachen» (Extrinsische Motivation) sowie je zwei Effekte im Profil «Wirtschaft/Recht» (Einstellung zur Gleichberechtigung der Geschlechter, Politisches Wissen) und «musisch» (Planungsstrategien, Politisches Interesse) - weisen ebenfalls auf die spezifische Bedeutsamkeit des Maturitätsprofils für die jungen Männer hin. Hinsichtlich der Entwicklung der Planungsstrategien und der Einstellung zur Gleichberechtigung der Geschlechter lassen sich diese Effekte für die jungen Männer positiv interpretieren. Vor allem bei der Entwicklung des politischen Wissens (Profil "Wirtschaft/Recht») und des politischen Interesses (musisches Profil) wird aber deutlich, dass sich der Lernkontext für die männlichen Jugendlichen auch negativ auf die Entwicklung auswirken kann.

Zeigen sich systematische Unterschiede zwischen weiblichen Jugendlichen eines Profils im Vergleich zu ihren Kolleginnen der anderen Profile, so können diese einzig im Bereich der politischen Kompetenzen identifiziert werden. Der Besuch des altsprachlichen Ausbildungsganges und des Profils "Wirtschaft/Recht» führt dazu, dass die jungen Frauen in stärkerem Ausmass als ihre Kolleginnen der anderen Profile ihr politisches Wissen weiterentwickelt haben, ohne dass sich vergleichbare Effekte bei den jungen Männern gezeigt hätten. In beiden Fällen waren zum Zeitpunkt des 10. Schuljahres noch keine geschlechtsspezifischen Effekte in Abhängigkeit des Maturitätsprofils sichtbar. Bei den Schülerinnen findet damit ein Angleichungsprozess an das höhere individuelle Kompetenzniveau ihrer anzahlmässig überrepräsentierten Kollegen im Profil statt. Im Gegensatz zu diesen beiden Profilen erweist sich für die Schülerinnen das neusprachlichen Maturitätsprofil für die Entwicklung des politischen Wissens als weniger produktiv, da die Schülerinnen trotz vergleichbarem Ausgangsniveau im 10. Schuljahr einen systematisch geringeren Entwicklungsverlauf aufweisen als ihre Kolleginnen der anderen Profile.

Im musischen Profil zeigt sich der Lernkontext nicht für die Schülerinnen, sondern für die Schüler als weniger funktional für die Entwicklung der politischen Kompetenzen. Hier weisen die Schüler eine systematisch geringere Interessensentwicklung im politischen Bereich auf als die Schüler der anderen Profile, ohne dass ein solcher Effekt auch bei den jungen Frauen zu beobachten wäre. Der oben beschriebene Angleichungsprozess zeigt sich hier in gegenläufiger 
Richtung als Annäherung an das durchschnittlich geringere politische Interesse auf individueller Ebene der anzahlmässig überrepräsentierten Kolleginnen im selben Profil.

Insgesamt lässt sich somit feststellen, dass die beiden Maturitätsprofile, die systematisch häufiger von Frauen besucht werden («Neue Sprachen», «musisch»), weniger funktional sind für die Entwicklung der politischen Kompetenzen. Beim neusprachlichen Profil sind systematische Effekte bei den Schülerinnen, beim musischen Profil bei den Schülern zu beobachten. Ebenfalls negative Effekte für die Schüler ergeben sich im Profil «Wirtschaft/Recht». Positive Effekte für die Schüler/innen zeigen sich hingegen in jenen Profilen, die mehrheitlich von Schülern gewählt werden ("Wirtschaft/Recht») oder in denen die Geschlechter zumindest entsprechend der Grundgesamtheit verteilt sind («Alte Sprachen»).

Die These, dass die Erklärung dieser Befunde entsprechend den Erfahrungen in der Arbeitswelt (Heintz et al., 1997) in der Verteilung der Geschlechter in den Profilen liegen könnte (These $2 \mathrm{~b}$ ), erhält damit nicht genügend Unterstützung, da sich trotz ähnlichem Geschlechterverhältnis im musischen und neusprachlichen Profil die Effekte unterscheiden. Zu vermuten ist, dass das spezifische fachlichen Profil der jeweiligen Schwerpunkte, die thematischen Bedeutungszuschreibungen durch die Lehrpersonen und Schüler/innen sowie weitere Prozesse und Dynamiken, die beispielsweise geprägt sind durch spezifische Rollenerwartungen oder Berufs- und Studienvorstellungen, die Entwicklung in stärkerem Masse beeinflussen (Eccles, 2005, 2006).

Warum sich Interaktionseffekte mit differenziellem Effekt für die jungen Frauen gehäuft im politischen Bereich zeigen, ist genauer zu untersuchen. Allenfalls könnten Wissensbestände in Kompetenzbereichen stärker über Maturitätsprofile differenziell beeinflusst sein als Einstellungen oder Selbstkonzepte. Allerdings müsste dies dann auch im Umweltbereich der Fall sein, in dem in dieser Studie ebenfalls Wissensfragen gestellt worden sind. Hier ergeben sich allerdings keine vergleichbaren Effekte. $\mathrm{Zu}$ analysieren ist damit, inwiefern sich diese beiden thematischen Bereiche voneinander unterscheiden. Eine Erklärung könnte in der Nähe zu den curricularen Vorgaben der in den Profilen unterrichteten Fächer liegen, wobei bei der Durchsicht der Schwerpunkte tendenziell eine grössere Nähe im polit-ökonomischen Bereich als im Umweltbereich wahrgenommen werden kann.

Einzig im Profil mit mathematisch-naturwissenschaftlichem Schwerpunkt, das häufiger von jungen Männern als von jungen Frauen gewählt wird, zeigen sich weder im 10. Schuljahr noch im Entwicklungsverlauf systematische Interaktionseffekte zwischen dem Maturitätsprofil und dem Geschlecht. Dieser Lernkontext erzeugt damit einzig einen profil-, aber keinen in Abhängigkeit des Maturitätsprofils stehenden geschlechtsspezifischen Einfluss auf die Kompetenzentwicklung. Dieser Befund unterstützt das oben formulierte Ergebnis, dass das numerische Verhältnis der Geschlechter innerhalb eines Lernkon- 
textes die Kompetenzentwicklung der Schüler und Schülerinnen in den entsprechenden Profilen im Vergleich zu den anderen Profilen nicht hinreichend erklären kann.

Abschliessend lässt sich festhalten, dass jedes einzelne Maturitätsprofil für die Kompetenzentwicklung der Schüler/innen einen je spezifischen Einflusskontext darstellt, welcher in Zukunft differenzierter untersucht werden sollte. So bleibt aufgrund der vorliegenden Ergebnisse offen, welche Aspekte des Lernkontextes die Entwicklungen effektiv beeinflusst haben. In Ergänzung zu den vorliegenden Analysen haben daher zukünftige Studien mehr das Interaktions- und Lernverhalten der Lehrpersonen und Schüler/innen in Abhängigkeit der fachlichen Schwerpunkte in den einzelnen Profilen, auch unter geschlechtsspezifischem Fokus, zu untersuchen, um genauere Erkenntnisse über das Wirkungs- und Bedingungsgefüge zu gewinnen. Aus methodischer Perspektive sind diese Analysen zu ergänzen mit mehrebenenanalytischen Untersuchungen, die die Klassenebene verstärkt in den Blick nehmen, da die Untersuchungsgruppe eine geschachtelte Struktur aufweist (Individuen, Klassen, Schulen). Zudem sind die teils explorativen Auswertungen, bedingt durch die grosse Anzahl an Indikatoren und dem noch wenig erforschten Untersuchungsgegenstand, weitergehend zu differenzieren.

\section{Anmerkungen}

1 Bundesamt für Statistik (2005). Statistik der Bildungsabschlüsse. $\mathrm{T}$ 15.2.2.1.2./ $\mathrm{T}$ 15.2.1.1.1.: http://www.bfs.admin.ch/bfs/portal/de/index/themen/15/04/00/blank/uebersicht.html (24.5.07)

2 Bildungsdirektion des Kantons Zürich, Bildungsplanung (2005). Statistikbereiche. http://www.bista.zh.ch/usi/us-bstak.aspx; (24.5.07)

3 Beispielitem: «Wie häufig führen Sie die folgenden Aktivitäten in Ihrer Freizeit durch? In der Freizeit arbeite ich aktiv in einem Verein oder einer Organisation» (Antwortformat: $1=1=$ nie,. .5 =sehr häufig)

\section{Literatur}

Artelt, C., Baumert, J., Julius-McElvany, N. \& Peschar, J. (2003). Das Lernen lernen. Voraussetzungen für lebensbegleitendes Lernen. Ergebnisse von PISA 2000. Paris: OECD.

Baumert, J., Roeder, P. M., Gruehn, S., Heyn, S., Köller, O., Rimmele, R., et al. (1996). Bildungsverläufe und psychosoziale Entwicklung im Jugendalter (BIJU). In K.-P. Treumann, G. Neubauer, R. Möller \& J. Abel (Hrsg.), Methoden und Andwendungen empirischer pädagogischer Forschung (S. 170-180). Münster: Waxman.

Baumert, J., Bos, W. \& Lehmann, R. (2000). TIMSS/III. Dritte Internationale Mathematikund Naturwissenschaftsstudie. Mathematische und naturwissenschaftliche Bildung am Ende der Schullaufbahn. Band 2: Mathematische und physikalische Kompetenzen am Ende der gymnasialen

Bieri, C. \& Forrer, E. (2005). Cool, kompetent und kein bisschen weise? Chur, Zürich: Rüegger Verlag.

Brühlwiler, C. \& Biedermann, H. (2005). Selbstreguliertes Lernen als Voraussetzung für erfolgreiches Mathematiklernen. In C. Zahner Rossier (Hrsg.), PISA 2003: Kompetenzen für die Zukunft. Zweiter nationaler Bericht (S. 57-73). Neuchâtel, Bern: BFS/EDK 
Bundesamt für Statistik (2003). Schweizerische Gesundheitsbefragung 2002. Erste Ergebnisse. Neuenburg: BFS.

Cohen, J. (1988). Statistical power analysis for the behavioral sciences. Hillsdale, New York: Erlbaum.

Eccles, J. S. (2005). Studying Gender and Ethnic Differences in Participation in Math, Physical Science, and Information Technology. In J. E. Jacobs \& S. D. Simpkins (Ed.), Leaks in the Pipeline to Math, Science and Technology Careers (p. 7-14). San Francisco: JosseyBass.

Eccles, J. S. (2006). Gendered Life Choices: Psychological Influences on Educational, Occupational, and Avocational Choices. Presentation May 11, 2006. Universität Zürich.

Eder, F. (1996). Das Schul- und Klassenklima. Innsbruck: Studien Verlag.

Epstein, S. (1993). Entwurf einer integrativen Persönlichkeitstheorie. In S. H. Filipp (Hrsg.), Selbstkonzeptforschung. Probleme, Befunde, Perspektiven (S. 15-46). Stuttgart: Klett-Cotta.

Fend, H. (1998a). Eltern und Freunde. Soziale Entwicklung im Jugendalter. Entwicklungspsychologie der Adoleszenz in der Moderne, Bd. 5. Bern: Hans Huber.

Fend, H. (1998b). Qualität im Bildungswesen. Schulforschung zu Systembedingungen, Schulprofilen und Lehrerleistung. Weinheim: Juventa.

Fend, H. (2000). Entwicklungspsychologie des Jugendalters (Vol. 1). Opladen: Leske+Budrich.

Filipp, S.-H. (2006). Entwicklung von Fähigkeitsselbstkonzepten. Kommentar zum Themenschwerpunkt. Zeitschrift für Pädagogische Psychologie 20 (1/2), 65-72.

Filipp, S. H. (Hrsg.). (1993). Selbstkonzeptforschung. Probleme, Befunde, Perspektiven. Stuttgart: Klett-Cotta.

Ford, M. E. (1982). Social cognition and social competence in adolescence. Developmental Psychology 18 (3), 323-340.

Gensicke, T. (2002). Individualität und Sicherheit in neuer Synthese? Wertorientierungen und gesellschaftliche Aktivität. In D. Shell (Hrsg.), Jugend 2002. Zwischen pragmatischem Idealismus und robustem Materialismus (S. 139-212). Frankfurt/Main: Fischer Taschenbuch.

Gildemeister, R. \& Wetterer, A. (1992). Wie Geschlechter gemacht werden. Die soziale Konstruktion der Zweigeschlechtlichkeit und ihre Reifizierung in der Frauenforschung. In G.A. Knapp \& A. Wetterer (Hrsg.), Traditionen Brüche. Entwicklungen feministischer Theorie (S. 201-254). Freiburg (Breisgau): Kore.

Greve, W. (Hrsg.). (2000). Psychologie des Selbst. Weinheim: Psychologie Verlags Union.

Grob, U. \& Maag Merki, K. (2001). Überfachliche Kompetenzen. Theoretische Grundlegung und empirische Erprobung eines Indikatorensystems. Bern: Peter Lang.

Grob, U., Maag Merki, K. \& Büeler, X. (2003). Young Adult Survey. Theoretische Begründung und empirische Befunde zur Validierung eines Indikatorensystems zu überfachlichen Kompetenzen. Schweizerische Zeitschrift für Bildungswissenschaften 25 (2), 309-330.

Heintz, B., Nadai, E., Fischer, R. \& Ummel, H. (1997). Ungleich unter Gleichen. Studien zur geschlechtsspezifischen Segregation des Arbeitsmarktes. Frankfurt a. M.: Campus.

Helmke, A. \& Weinert, F. E. (1997). Bedingungsfaktoren schulischer Leistungen. In F. E. Weinert (Hrsg.), Psychologie des Unterrichts und der Schule. Enzyklopädie der Psychologie, Pädagogische Psychologie, Bd. 3 (S. 71-176). Göttingen: Hogrefe.

Horstkemper, M. (1991). Schule, Geschlecht und Selbstvertrauen. Eine Längsschnittstudie über Mädchensozialisation in der Schule. Weinheim: Juventa.

Hurrelmann, K., Klocke, A., Melzer, W. \& Ravens-Sieberer, U. (Hrsg.). (2003). Jugendgesundheitssurvey. Internationale Vergleichsstudie im Auftrag der Weltgesundheitsorganisation WHO. München: Juventa.

Köller, O., Watermann, R. \& Trautwein, U. (Hrsg.). (2004). Wege zur Hochschulreife in Baden-Württemberg. TOSCA - Eine Untersuchung an allgemein bildenden und beruflichen Gymnasien. Opladen: Leske+Budrich.

Lehmann, R. H., Hunger, S., Ivanova, S., Gänsfuss, R. \& Hoffmann, E. M. (2004). LAU 11. Aspekte der Lernausgangslage und der Lernentwicklung Klassenstufe 11. Ergebnisse einer läng- 
sschnittlichen Untersuchung in Hamburg. Hamburg: Behörde für Bildung und Sport, Amt für Schule.

Maag Merki, K. (Hrsg.) (2006a). Lernort Gymnasium. Individuelle Entwicklungsverläufe und Schulerfahrungen. Bern: Haupt Verlag.

Maag Merki, K. (2006b). Gesamtdiskussion. In K. Maag Merki (Hrsg.), Lernort Gymnasium. Individuelle Entwicklungsverläufe und Schulerfahrungen. (S. 177-194). Bern: Haupt Verlag.

Maag Merki, K. \& Leutwyler, B. (2004). Evaluation Mittelschulen - Überfachliche Kompetenzen. Zwischenbericht der zweiten Erhebung 2004. Zürich: Forschungsbereich Schulqualität \& Schulentwicklung, Pädagogisches Institut, Universität Zürich.

Rakoczy, K. (2005). Learning Enviroment and Learning Motivation in Mathematics Instruction. Differential Relations depending on the Topic? Paper presented on the Congress of the European Association for Research on Learning and Instruction (EARLI) in Nicosia, Cyprus, 23.-27. August 2005.

Ramseier, E. (2004). Motivation als Ergebnis und als Determinante schulischen Lernens. Zürich: Zentralstelle der Studentenschaft.

Ramseier, E., Allraum, J. \& Stadler, U. (2005). Neue Fächerstruktur und Ausbildungserfolg. In E. Ramseier, F. Grin, S. Müller, R. Alliata, J. Allraum, E. Dozio, P. Labudde, K. Maag Merki, E. Pagnossin, U. Stadler, E. Stocker \& I. Willimann (Hrsg.), Evaluation der Maturitätsreform 1995 (EVAMAR). Neue Fächerstruktur - Pädagogische Ziele - Schulentwicklung. Schlussbericht zur Phase 1 (S. 45-155). Bern: EDI, Staatssekretariat für Bildung und Forschung SBF.

Sandmeier, A. \& Maag Merki, K. (2006). Politische Kompetenzen. In K. Maag Merki (Hrsg.), Lernort Gymnasium. Individuelle Entwicklungsverläufe und Schulerfahrungen (S. 159-174). Bern: Haupt Verlag.

Schmid, C. (2003). Fördert der Schulunterricht an Gymnasien das politische Interesse von Jugendlichen? Zeitschrift für Soziologie der Erziehung und Sozialisation, 23 (4), 371-384.

Schweizerischer Bundesrat \& Erziehungsdirektorenkonferenz EDK. (1995). Verordnung des Bundesrates/Reglement der EDK über die Anerkennung von gymnasialen Maturitätsausweisen (MAR) vom 16. Januar/15. Februar 1995. Bern: Schweizerischer Bundesrat/EDK.

Stanat, P., \& Kunter, M. (2001). Kooperation und Kommunikation. In Deutsches PISA-Konsortium (Hrsg.), PISA 2000. Basiskompetenzen von Schülerinnen und Schülern im internationalen Vergleich (S. 300-322). Opladen: Leske+Budrich.

Trautwein, U. \& Lüdtke, O. (2004). Aspekte von Wissenschaftspropädeutik und Studierfähigkeit. In O. Köller, R. Watermann, U. Trautwein \& O. Lüdtke (Hrsg.), Wege zur Hochschulreife in Baden-Württemberg. TOSCA - Eine Untersuchung an allgemein bildenden und beruflichen Gymnasien (S. 327-366). Opladen: Leske+Budrich.

Watermann, R., Nagy, G. \& Köller, O. (2004). Mathematikleistungen in allgemein bildenden und beruflichen Gymnasien. In O. Köller, R. Watermann, U. Trautwein \& O. Lüdtke (Hrsg.), Wege zur Hochschulreife in Baden-Württemberg. TOSCA - Eine Untersuchung an allgemein bildenden und beruflichen Gymnasien (S. 205-283). Opladen: Leske+Budrich.

Weinert, F. E. (2001). Concept of Competence: A Conceptual Clarification. In D. S. Rychen \& L. H. Salganik (Hrsg.), Defining and Selecting Key Competencies (S. 45-65). Seattle, Bern: Hogrefe \& Huber Publishers.

Wigfield, A., Byrnes, J. P. \& Eccles, J. S. (2006). Development during early and middle adolescence. In P. A. Alexander \& P. H. Winne (Hrsg.), Handbook of Educational Psychology. Second Edition (S. 87-113). London: Lawrence Earlbaum Associates.

Zinnecker, J. (1975). Der heimliche Lehrplan: Untersuchungen zum Schulunterricht. Weinheim: Beltz.

Schlagworte: Überfachliche Kompetenzen, Entwicklung, Gymnasium, Effektivität von Lernkontexten 


\title{
Les profils de la maturité (baccalauréat) comme contextes d'apprentissage différenciés : Une analyse empirique des relations entre cadres institutionnels et développement de compétences des gymnasiens et gymnasiennes.
}

\begin{abstract}
Résumé
Létude présentée dans cette contribution examine dans quelle mesure le développement de différentes compétences transversales au cours de la formation gymnasiale dépend du genre des étudiant-e-s et du contexte d'apprentissage spécifique au profil de la maturité suivie. Les données recueillies sont soumises à des analyses de la variance sur la base d'un plan factoriel $2 \times 2$. Les résultats montrent que les profils de la maturité, comme institutionnalisation de la formation scolaire, entretiennent une relation significative avec le développement de compétences transversales des étudiant-e-s. Des effets d'interaction sont significatives sur certaines dimensions particulières de compétence; ils indiquent que sur la plupart des dimensions de compétence les étudiants masculins d'un profil de maturité donné se démarquent plus de leurs collègues d'autres profils que les étudiantes.
\end{abstract}

Mots clés: Compétences transversales, étude de genre, effet de la formation, enseignement secondaire, profil de maturité.

\section{Profili di maturità: contesti di apprendimento differenziali. Un'analisi empirica del rapporto tra i quadri istituzionali e lo sviluppo di competenze dei liceali.}

\section{Riassunto}

In questo articolo è posta la domanda su come - nello sviluppo di competenze transdisciplinari nella formazione liceale - siano percettibili gli effetti sia specifici ai profili, sia specifici alle differenze di genere in relazione al contesto di apprendimento (profili di maturità). I risultati presentati si basano sull'analisi della covarianza multifattoriale $(2 \times 2)$ univariata. Tali risultati dimostrano come i profili di maturità, in quanto istituzionalizzazione della formazione scolastica, hanno un rapporto significativo con lo sviluppo di competenze transdisciplinari degli studenti. Gli effetti di interazione sono identificabili in singole dimensioni di competenza e, nella maggior parte dei casi, dimostrano che in alcune materie, i ragazzi si distinguono rispetto ai loro colleghi con altri profili, mentre le ragazze meno.

Parole chiave: competenze trasversali, studi di genere, impatto della formazione, insegnamento secondario, profilo di maturità. 


\section{Maturity profiles as differential learning contexts : An empirical analysis of the relation between institutional frameworks and competency development in male and female students attending Gymnasium (upper secondary school).}

\section{Summary}

«Maturity profiles» are different upper secondary school tracks diplomas. This paper investigates to what extent the development of cross-curricular competencies in upper secondary education depends on the students gender and on their learning context related to each maturity profile. The results presented are based on a $2 \times 2$ analysis of variance. The results show that maturity profiles, as institutionalisation of secondary schooling, show a significant correlation with the development of students' cross-curricular competencies in students. Interaction effects can be identified on specific competency dimensions; in most cases they indicate stronger differences of competency between young males of different maturity profiles than between young females.

Keywords: cross-curricular competencies, development, upper secondary school, High school, learning contexts efficiency, gender. 
T h e m a 